\title{
The National Diabetic Retinopathy Laser Treatment Audit. III. Clinical outcomes
}

Abstract

Purpose To describe the short-term clinical outcomes for a cohort of patients undergoing first photocoagulation treatment for proliferative retinopathy or maculopathy in the United Kingdom.

Method Nine-month follow-up of the Royal College of Ophthalmologists' national audit of laser treatment for diabetic retinopathy. Results For eyes with maculopathy, 9.2\% had had a deterioration in visual acuity equivalent to a doubling of the visual angle and $3.3 \%$ of eyes had a visual acuity of less than $6 / 60$ at follow-up. There had been an improvement in the macular oedema or exudate in $64.6 \%$ and 77.3\% respectively. Prognostic factors for a poorer visual acuity at follow-up were worse visual acuity at baseline, the presence of diffuse (vs focal) oedema and grid (vs focal) treatment. For eyes with proliferative retinopathy, the retinal neovascularisation had regressed fully in $50.8 \%$ of cases, whilst there had been no change or a deterioration in $10.3 \%$. A visual acuity of less than $6 / 60$ at follow-up was present in $8.6 \%$ of eyes. There was a poor morphological outcome at followup (as defined by rubeosis, new tractional detachment or having had a vitrectomy) in $\mathbf{7 . 2} \%$. Risk factors for poor morphological outcome were the presence of 'high-risk characteristics', female sex and the presence of concurrent maculopathy at baseline.

Regression of neovascularisation was associated with greater areas of retinal ablation at the initial treatment session. Although some eyes with proliferative retinopathy appeared to be undertreated initially compared with DRS and ETDRS protocols, some of these eyes did respond to lower amounts of treatment.

Conclusion For maculopathy, poorer outcome was related to worse visual acuity at baseline, diffuse (vs focal) maculopathy, and grid treatment. For proliferative retinopathy, poorer outcome was related to 'high-risk characteristics' and coexistence of maculopathy at baseline, and improvement was related to larger areas of ablation. The relationship of poor outcome with worse initial disease argues for earlier detection of retinopathy.
Key words Diabetic retinopathy, Laser, Maculopathy, Photocoagulation, Proliferative retinopathy

Despite the availability of an effective treatment, diabetic retinopathy remains a leading cause of blindness in the western world. ${ }^{1-9}$ Whether the Saint Vincent Declaration target of reducing the incidence of blindness due to diabetes by one-third or more can be achieved depends on a number of factors including screening and the efficacy of treatment. ${ }^{10}$ This audit was designed to examine the processes of delivery of photocoagulation treatment for diabetic retinopathy throughout the United Kingdom. This paper reports on the short-term clinical outcomes for a national cohort of diabetic patients undergoing first laser treatment for proliferative retinopathy or maculopathy.

\section{Materials and methods}

The full description of the methodology for this audit is given elsewhere. ${ }^{11}$ In brief, all Consultant Ophthalmologists in the United Kingdom who undertake laser treatment for diabetic retinopathy were invited to participate. Eligible for inclusion were those patients undergoing first laser treatment for proliferative retinopathy or maculopathy during June and July 1995. Baseline questionnaires were completed by the ophthalmologist performing the laser treatment. For the purpose of this study, maculopathy was defined as exudative where exudate was the predominant feature, oedematous where oedema was the predominant feature, and ischaemic where there was actual or presumed widespread capillary closure. Oedematous maculopathy was subdivided into that due to focal or multifocal leakage (where the oedema was associated with multiple visible capillary abnormalities), and oedema due to diffuse leakage (where no, or very few such abnormalities, were visible). The term focal maculopathy was used to describe both exudative and focal/multifocal oedematous maculopathy.

\author{
C.C. Bailey \\ J.M. Sparrow \\ R.H.B. Grey \\ $\mathrm{H}$. Cheng \\ The Royal College of \\ Ophthalmologists \\ London, UK
}

Miss Clare Bailey, MRCP, FRCOphth

The Royal College of Ophthalmologists

17 Cornwall Terrace London NW1 4QW, UK

Tel: +44 (0)1719350702

This project was funded by the Department of Health

Received: 18 May 1998 Accepted in revised form: 15 January 1999 
Follow-up questionnaires were completed as close as possible to 9 months following the initial laser treatment. The follow-up questionnaire contained questions on change in retinopathy features, visual acuity, and the amount and type of treatment given.

All the data were returned to the Royal College of Ophthalmologists and were double-entered, stored on a computerised database, and analysed using a commercially available statistical software package (SPSS for windows 6.0).

\section{Results}

\section{Maculopathy}

Follow-up questionnaires were returned for $85 \%$ (464) of the 546 patients initially recruited. Of these, 25 (5.4\%) patients were lost to follow-up and 13 (2.8\%) had died. Follow-up data were collected at a median of 277 days (99-553 days, SD 59.4). There was no significant difference in the age, gender, type of maculopathy or visual acuity in the treated eye at baseline between those with and without follow-up data.

\section{Visual acuity}

The best-recorded visual acuities at follow-up compared with baseline are shown in Table 1 . At follow-up 31.1\% (132) of eyes had a visual acuity of $6 / 6$ or better, and $16.7 \%(71)$ had a visual acuity of $6 / 24$ or worse. Where the visual acuity was less than $6 / 9$ at follow-up this was said to be due to maculopathy in $74.1 \%$, due to cataract in $9.9 \%$, due to a combination of cataract and maculopathy in $12.3 \%$, due to vitreous haemorrhage in $1.2 \%$ and due to macular traction in $1.2 \%$. Two eyes had disciform agerelated macular degeneration and two eyes were amblyopic. Where the visual acuity in the treated eye was less than $6 / 60$ at follow-up $(n=14)$ this was said to be due to cataract in $7.1 \%$, vitreous haemorrhage in $7.1 \%$, disciform age-related macular degeneration in $7.1 \%$, and maculopathy in $78.6 \%$. At follow-up there was no change ( \pm 1 Snellen line) in the best-recorded visual acuity in $79.6 \%$, with a deterioration of more than 1 line in $10.1 \%$ and an improvement of more than 1 line in $10.3 \%$. In order for a comparison to be made with other literature, the Snellen visual acuities were converted to their $\log$ MAR equivalent; $9.2 \%$ of treated eyes lost $0.3 \log$ units or more of visual acuity (equivalent to a loss of 3 lines or more on the ETDRS logMAR chart, or a doubling of the visual angle ${ }^{8}$ ).

Table 2 shows the visual acuity at follow-up based upon the type of maculopathy at baseline. For those with exudative maculopathy prior to treatment, by the time of follow-up there had been no change ( \pm 1 Snellen line) in $83.2 \%$, and an improvement of more than 1 line in $7.6 \%$. There had been a deterioration of $0.3 \log$ units or more in $8.5 \%$. For those with oedematous maculopathy, at followup there had been no change ( \pm 1 Snellen line) in $71.2 \%$, and an improvement of more than 1 line in $14.3 \%$. There had been a deterioration of $0.3 \log$ units or more in $15.4 \%$. For those with diffuse oedematous maculopathy prior to treatment (a subgroup of those with oedematous maculopathy), the visual acuity at follow-up was $6 / 9$ or better in $13.2 \%$ (5) and $6 / 24$ or worse in $52.6 \%$ (20). At follow-up, there had been no change in vision $( \pm 1$ Snellen line) in $71.8 \%$, and an improvement of more than 1 line in $15.1 \%$. There had been a deterioration of $0.3 \mathrm{log}$ units or more in $28.9 \%$.

Stepwise multiple linear regression analysis was performed to determine prognostic factors for visual outcome. Factors included in the anlaysis were: visual

Table 1. Best-recorded visual acuity at follow-up compared with baseline for eyes undergoing photocoagulation treatment for maculopathy (row percentages)

\begin{tabular}{|c|c|c|c|c|c|c|c|c|c|c|c|}
\hline \multirow{2}{*}{$\begin{array}{l}\text { Visual acuity } \\
\text { at baseline }\end{array}$} & \multicolumn{11}{|c|}{ Visual acuity at follow-up } \\
\hline & & $6 / 5$ & $6 / 6$ & $6 / 9$ & $6 / 12$ & $6 / 18$ & $6 / 24$ & $6 / 36$ & $6 / 60$ & $<6 / 60$ & $\mathrm{All}^{\mathrm{a}}$ \\
\hline \multirow[t]{2}{*}{$6 / 5$} & $n$ & 26 & 14 & 4 & 0 & 0 & 1 & 0 & 0 & 0 & 45 \\
\hline & $\%$ & $57.8 \%$ & $3.1 \%$ & $8.9 \%$ & & & $1.2 \%$ & & & & $10.6 \%$ \\
\hline \multirow[t]{2}{*}{$6 / 6$} & $n$ & 11 & 46 & 30 & 6 & 2 & 2 & 1 & 0 & 0 & 98 \\
\hline & $\%$ & $11.2 \%$ & $46.9 \%$ & $30.6 \%$ & $6.1 \%$ & $2.0 \%$ & $2.0 \%$ & $1.0 \%$ & & & $23.1 \%$ \\
\hline \multirow[t]{2}{*}{$6 / 9$} & $n$ & 7 & 17 & 55 & 17 & 3 & 2 & 2 & 1 & 1 & 105 \\
\hline & $\%$ & $6.7 \%$ & $16.2 \%$ & $52.4 \%$ & $16.2 \%$ & $2.9 \%$ & $1.9 \%$ & $1.9 \%$ & $1.0 \%$ & $1.0 \%$ & $24.8 \%$ \\
\hline \multirow[t]{2}{*}{$6 / 12$} & $n$ & 0 & 7 & 15 & 23 & 11 & 0 & 3 & 2 & 1 & 62 \\
\hline & $\%$ & & $11.3 \%$ & $24.2 \%$ & $37.1 \%$ & $17.7 \%$ & & $4.8 \%$ & $3.2 \%$ & $1.6 \%$ & $14.6 \%$ \\
\hline \multirow[t]{2}{*}{$6 / 18$} & $n$ & 0 & 1 & 15 & 8 & 14 & 8 & 1 & 2 & 1 & 50 \\
\hline & $\%$ & & $2.0 \%$ & $30 \%$ & $16 \%$ & $28 \%$ & $16 \%$ & $2.0 \%$ & $4.0 \%$ & $2.0 \%$ & $11.8 \%$ \\
\hline \multirow[t]{2}{*}{$6 / 24$} & $n$ & 1 & 1 & 0 & 5 & 7 & 6 & 4 & 1 & 5 & 30 \\
\hline & $\%$ & $3.3 \%$ & $3.3 \%$ & & $16.7 \%$ & $23.3 \%$ & $20.0 \%$ & $13.3 \%$ & $3.3 \%$ & $16.7 \%$ & $7.1 \%$ \\
\hline \multirow[t]{2}{*}{$6 / 36$} & $n$ & 0 & 1 & 1 & 2 & 1 & 5 & 2 & 5 & 3 & 20 \\
\hline & $\%$ & & $5.0 \%$ & $5.0 \%$ & $10.0 \%$ & $5.0 \%$ & $25.0 \%$ & $10.0 \%$ & $25.0 \%$ & $15.0 \%$ & $4.7 \%$ \\
\hline \multirow[t]{2}{*}{$6 / 60$} & $n$ & 0 & 0 & 1 & 0 & 0 & 0 & 1 & 6 & 0 & 8 \\
\hline & $\%$ & & & $12.5 \%$ & & & & $12.5 \%$ & $75.0 \%$ & & $1.9 \%$ \\
\hline \multirow[t]{2}{*}{$<6 / 60$} & $n$ & 0 & 0 & 0 & 0 & 1 & 0 & 1 & 1 & 3 & 6 \\
\hline & $\%$ & & & & & $16.7 \%$ & & $16.7 \%$ & $16.7 \%$ & $50 \%$ & $1.4 \%$ \\
\hline \multirow[t]{2}{*}{ All } & $n$ & 45 & 87 & 121 & 61 & 39 & 24 & 15 & 18 & 14 & 424 \\
\hline & $\%$ & $10.6 \%$ & $20.5 \%$ & $28.5 \%$ & $14.4 \%$ & $9.2 \%$ & $5.7 \%$ & $3.5 \%$ & $4.2 \%$ & $3.3 \%$ & $100 \%$ \\
\hline
\end{tabular}

Missing baseline visual acuity data in 2 cases.

${ }^{a}$ Column percentage. 
Table 2. The visual acuity at follow-up based upon the type of maculopathy at baseline

\begin{tabular}{|c|c|c|c|c|}
\hline \multirow[b]{2}{*}{ Visual acuity at follow-up } & \multicolumn{2}{|c|}{ Exudative maculopathy } & \multicolumn{2}{|c|}{ Oedematous maculopathy } \\
\hline & $n$ & $\%$ & $n$ & $\%$ \\
\hline $6 / 5$ & 43 & $13.8 \%$ & 2 & $2.0 \%$ \\
\hline $6 / 6$ & 72 & $23.2 \%$ & 13 & $12.7 \%$ \\
\hline $6 / 9$ & 97 & $31.2 \%$ & 20 & $19.6 \%$ \\
\hline $6 / 12$ & 43 & $13.8 \%$ & 16 & $15.7 \%$ \\
\hline $6 / 18$ & 24 & $7.7 \%$ & 14 & $13.7 \%$ \\
\hline $6 / 24$ & 15 & $4.8 \%$ & 9 & $8.9 \%$ \\
\hline $6 / 36$ & 3 & $1 \%$ & 11 & $10.8 \%$ \\
\hline $6 / 60$ & 7 & $2.3 \%$ & 10 & $9.8 \%$ \\
\hline$<6 / 60$ & 7 & $2.3 \%$ & 7 & $6.9 \%$ \\
\hline Total & 311 & $100 \%$ & 102 & $100 \%$ \\
\hline
\end{tabular}

acuity prior to treatment, age and gender of the patient, type and duration of diabetes, type of maculopathy (focal/diffuse or exudative/oedematous), type of treatment given (focal or grid), systematic screening to detect the retinopathy, grade of ophthalmologist performing the treatment and the waiting times for treatment (from listing and from referral).

The visual acuity at follow-up was significantly related to the visual acuity at baseline (slope $=0.68, p<0.01$ ). Focal maculopathy was associated with better visual acuity at follow-up than was diffuse maculopathy (average logMAR difference $=0.14, p<0.01$ ) and focal treatment was associated with a better visual acuity at follow-up compared with grid treatment (average $\operatorname{logMAR}$ difference $=0.15, p<0.01$ ). Other factors were not independently related to the visual acuity at follow-up.

The change in acuity from baseline to follow-up was related to the visual acuity at baseline: those eyes with worse visual acuity at baseline had less deterioration in visual acuity by the time of follow-up (slope $=-0.28$, $p<0.01$ ). Diffuse maculopathy was associated with more deterioration in visual acuity than focal maculopathy (average logMAR difference in deterioration $=0.03$, $p<0.01$ ), and grid treatment was associated with more deterioration in visual acuity than focal treatment (average $\log$ MAR difference in deterioration $=0.22, p<0.01$ ). Other factors were not independently related to the change in visual acuity between baseline and follow-up.

Multiple logistic regression analysis showed that a loss of $0.3 \log$ units of visual acuity (doubling of the visual angle) or more was associated with grid (vs focal) treatment (odds ratio $(\mathrm{OR})=4.2, p<0.01$ ), and diffuse (vs focal) maculopathy ( $\mathrm{OR}=4.8, p=0.02)$, but not independently related to other factors.

\section{Change in retinopathy features}

Table 3 shows the change in macular exudate or oedema at the time of follow-up. These findings were based upon clinical examination alone in $84 \%$, aided by colour photographs in $10.7 \%$, and by fluorescein angiography in $5.3 \%$. Logistic regression analysis found that a morphological improvement in the maculopathy was more common with better visual acuity at baseline $(p<0.01)$. Although a morphological improvement was more common in eyes with exudative (vs oedematous) maculopathy ( $p=0.02)$ and in eyes undergoing focal (vs grid) treatment $(p=0.02)$, this was not significant when allowing for the visual acuity at baseline.

Progression to proliferative retinopathy

Overall for the eyes with maculopathy at baseline, 7.1\% (30) had developed proliferative retinopathy by the time of follow-up. Of eyes with focal maculopathy at baseline $6.3 \%$ (23) developed proliferative retinopathy by the time of follow-up, compared with $13.5 \%$ (5) of eyes with diffuse oedematous maculopathy at baseline (Fisher's exact test, $p=0.09$ ).

\section{Treatment given}

Focal treatment alone had been given to $76.3 \%$ (325) of patients, grid treatment alone to $14.8 \%$ (63) and a combination of focal and grid treatment to $8.9 \%$ (38). At the time of follow-up, the eye had been given only one treatment session in $278(65.2 \%)$, there had been two

Table 3. The change in maculopathy features at follow-up

\begin{tabular}{lrc}
\hline & $n$ & $\%$ \\
\hline Change in macular exudate $^{\mathrm{a}}$ & & \\
Improved & 232 & $77.3 \%$ \\
Not changed & 28 & $9.3 \%$ \\
Deteriorated & 35 & $11.7 \%$ \\
Not known & 5 & $\frac{1.7 \%}{100 \%}$ \\
\cline { 2 - 2 } Total & 300 & \\
Change in macular oedema ${ }^{\mathrm{b}}$ & & \\
Improved & 62 & $64.6 \%$ \\
Not changed & 22 & $22.9 \%$ \\
Deteriorated & 7 & $7.3 \%$ \\
Not known & 5 & $5.2 \%$ \\
Total & 96 & $100 \%$ \\
Change in diffuse macular oedema & \\
Improved & & \\
Not changed & 23 & $65.7 \%$ \\
Deteriorated & 7 & $20.0 \%$ \\
Not known & 3 & $8.6 \%$ \\
Total & 2 & $5.7 \%$ \\
\cline { 2 - 2 } & 35 & $100 \%$ \\
\hline
\end{tabular}

${ }^{a}$ For eyes with predominantly exudative maculopathy at baseline. Missing data in 11 çases.

${ }^{b}$ For eyes with predominantly oedematous maculopathy at baseline. Missing data in 6 cases.

${ }^{c}$ This represents a subgroup of those with macular oedema. Missing data in 3 cases. 
treatment sessions in $109(25.6 \%)$, three sessions in 31 $(7.3 \%)$, and four or more sessions in $1.9 \%$ (8) (maximum 7 sessions).

For those eyes with exudative maculopathy at baseline, focal treatment had been given in $88.1 \%$ (274), grid treatment alone in $4.2 \%$ (13), and both grid and focal treatment in $7.7 \%$ (24). Where the exudates had improved at follow-up and no proliferative retinopathy had developed $(n=209)$ the median number of burns was 52 (range 4-1130, SD 155).

For eyes with focal or multifocal oedematous maculopathy at baseline, focal treatment had been given in $56.2 \%$ (36), grid treatment alone in $26.6 \%$ (17) and a combination of grid and focal treatment in $17.2 \%$ (11). Where the oedema had improved and no proliferative retinopathy had developed, the median number of burns was 118 (range 8-1226, SD 247).

For eyes with diffuse oedematous maculopathy at baseline, focal treatment alone had been given in $28.9 \%$ (11), grid treatment alone in $65.7 \%$ (25) and both grid and focal treatment in $5.3 \%$ (2). For those eyes where the diffuse oedema had improved and proliferative retinopathy had not developed, the median number of burns given was 125 (44-3218, SD 647).

The total number of laser burns was not related to the type of maculopathy at baseline, but significantly more burns were used for eyes treated with grid laser than eyes with just focal or focal and grid treatment $(p<0.01$, Mann-Whitney $U$ ).

\section{Retinopathy features in the other eye}

Maculopathy was said to be present in the other eye in $63.6 \%$ (269) of patients, whilst $5.9 \%$ (25) had proliferative retinopathy in the other eye. The other eye had received photocoagulation treatment in 53.2\% (225). In the better eye, $72.8 \%$ of patients had a visual acuity of $6 / 9$ or better, and $0.7 \%$ had a visual acuity of less than $6 / 60$.

\section{Proliferative retinopathy}

Follow-up questionnaires were returned for $82 \%$ (233) of the 284 patients with proliferative retinopathy initially recruited. Of these, $19(8.2 \%)$ were lost to follow-up and 5 $(2.1 \%)$ had died. Follow-up data were collected at a median of 273 days (range $76-564$ days, SD 56.6). There was no significant difference in the age, gender, bestrecorded visual acuity in the treated eye or the retinopathy features at baseline between those with and without follow-up data.

\section{Visual acuity}

The best-recorded visual acuities at follow-up compared with baseline are shown in Table 4 . At follow-up, 26.3\% of eyes had a visual acuity of $6 / 6$ or better, and $21.9 \%$ had a visual acuity of $6 / 24$ or worse. Where the visual acuity in the treated eye was less than $6 / 9$ this was said to be due to maculopathy in $45.3 \%$ (43), cataract in $17.9 \%$ (17), vitreous haemorrhage in $16.8 \%$ (16), both cataract and maculopathy in $9.5 \%$ (9), rubeotic glaucoma in $4.2 \%$ (4), and both maculopathy and traction detachment in $5.3 \%(5)$. For those eyes with a best-recorded visual acuity of less than $6 / 60$ at follow-up $(n=18)$, this was said to be due to maculopathy in $50 \%$ (9), vitreous haemorrhage in $11.1 \%$ (2), rubeotic glaucoma in $22.2 \%$ (4), and both maculopathy and traction detachment in $16.7 \%$ (3).

Stepwise multiple linear regression analysis showed that poorer visual acuity at follow-up was related to poorer visual acuity at baseline (slope $=0.8, p<0.01$ ), female sex (average logMAR difference $=0.1, p<0.01$ ) and the presence of maculopathy at baseline (average

Table 4. Best-recorded visual acuity at follow-up compared with baseline for eyes undergoing panretinal photocoagulation for proliferative retinopathy (row percentages)

\begin{tabular}{|c|c|c|c|c|c|c|c|c|c|c|c|}
\hline \multirow{2}{*}{$\begin{array}{l}\text { Visual acuity } \\
\text { at baseline }\end{array}$} & \multicolumn{11}{|c|}{ Visual acuity at follow-up } \\
\hline & & $6 / 5$ & $6 / 6$ & $6 / 9$ & $6 / 12$ & $6 / 18$ & $6 / 24$ & $6 / 36$ & $6 / 60$ & $<6 / 60$ & $\mathrm{All}^{\mathrm{a}}$ \\
\hline \multirow[t]{2}{*}{$6 / 5$} & $n$ & 8 & 13 & 8 & 0 & 0 & 0 & 0 & 0 & 0 & 29 \\
\hline & $\%$ & $27.6 \%$ & $44.8 \%$ & $27.6 \%$ & & & & & & & $13.9 \%$ \\
\hline \multirow[t]{2}{*}{$6 / 6$} & $n$ & 6 & 11 & 16 & 4 & 2 & 1 & 0 & 0 & 1 & 41 \\
\hline & $\%$ & $14.6 \%$ & $26.8 \%$ & $39 \%$ & $9.8 \%$ & $4.9 \%$ & $2.4 \%$ & & & $2.4 \%$ & $19.6 \%$ \\
\hline \multirow[t]{2}{*}{$6 / 9$} & $n$ & 2 & 10 & 28 & 8 & 0 & 2 & 2 & 1 & 1 & 54 \\
\hline & $\%$ & $3.7 \%$ & $18.5 \%$ & $51.9 \%$ & $14.8 \%$ & & $3.7 \%$ & $3.7 \%$ & $1.9 \%$ & $1.9 \%$ & $25.8 \%$ \\
\hline \multirow[t]{2}{*}{$6 / 12$} & $n$ & 3 & 1 & 6 & 14 & 6 & 0 & 1 & 0 & 0 & 31 \\
\hline & $\%$ & $9.7 \%$ & $3.2 \%$ & $19.4 \%$ & $45.2 \%$ & $19.4 \%$ & & $3.2 \%$ & & & $14.8 \%$ \\
\hline \multirow[t]{2}{*}{$6 / 18$} & $n$ & 0 & 1 & 1 & 8 & 2 & 3 & 2 & 4 & 1 & 22 \\
\hline & $\%$ & & $4.5 \%$ & $4.5 \%$ & $36.4 \%$ & $9.1 \%$ & $13.6 \%$ & $9.1 \%$ & $18.2 \%$ & $4.5 \%$ & $10.5 \%$ \\
\hline \multirow[t]{2}{*}{$6 / 24$} & $n$ & 0 & 0 & 0 & 2 & 1 & 5 & 0 & 0 & 2 & 10 \\
\hline & $\%$ & & & & $20 \%$ & $10 \%$ & $50 \%$ & & & $20 \%$ & $4.8 \%$ \\
\hline \multirow[t]{2}{*}{$6 / 36$} & $n$ & 0 & 0 & 0 & 1 & 0 & 1 & 1 & 1 & 2 & 6 \\
\hline & $\%$ & & & & $16.7 \%$ & & $16.7 \%$ & $16.7 \%$ & $16.7 \%$ & $33.3 \%$ & $2.9 \%$ \\
\hline \multirow[t]{2}{*}{$6 / 60$} & $n$ & 0 & 0 & 0 & 0 & 0 & 0 & 0 & 2 & 1 & 3 \\
\hline & $\%$ & & & & & & & & $66.7 \%$ & $33.3 \%$ & $1.4 \%$ \\
\hline \multirow[t]{2}{*}{$<6 / 60$} & $n$ & 0 & 0 & 0 & 1 & 0 & 0 & 1 & 1 & 10 & 13 \\
\hline & $\%$ & & & & $7.7 \%$ & & & $7.7 \%$ & $7.7 \%$ & $76.9 \%$ & $6.2 \%$ \\
\hline \multirow[t]{2}{*}{ All } & $n$ & 19 & 36 & 59 & 38 & 11 & 12 & 7 & 9 & 18 & 209 \\
\hline & $\%$ & $9.1 \%$ & $17.2 \%$ & $28.2 \%$ & $18.2 \%$ & $5.3 \%$ & $5.7 \%$ & $3.3 \%$ & $4.3 \%$ & $8.6 \%$ & $100 \%$ \\
\hline
\end{tabular}

${ }^{\mathrm{a}}$ Column percentage. 
Table 5. Change in the retinal new vessel features at follow-up for eyes with proliferative retinopathy prior to treatment

\begin{tabular}{|c|c|c|c|c|c|c|c|c|}
\hline \multirow{2}{*}{$\begin{array}{l}\text { Change to new } \\
\text { vessel features }\end{array}$} & \multicolumn{2}{|c|}{ All cases* } & \multicolumn{2}{|c|}{ NVD alone } & \multicolumn{2}{|c|}{ NVE alone } & \multicolumn{2}{|c|}{ NVD and NVE } \\
\hline & $n$ & $\%$ & $n$ & $\%$ & $n$ & $\%$ & $n$ & $\%$ \\
\hline Disappeared completely & 78 & $38.4 \%$ & 23 & $33.8 \%$ & 46 & $50 \%$ & 9 & $20.9 \%$ \\
\hline Regressed but still present & 80 & $39.4 \%$ & 39 & $57.3 \%$ & 22 & $23.9 \%$ & 19 & $44.2 \%$ \\
\hline Inactive fibrotic stalk & 24 & $11.8 \%$ & 4 & $5.9 \%$ & 12 & $13.9 \%$ & 8 & $18.6 \%$ \\
\hline Not changed & 10 & $4.9 \%$ & 1 & $1.5 \%$ & 7 & $7.6 \%$ & 2 & $4.6 \%$ \\
\hline Deteriorated & 11 & $5.4 \%$ & 1 & $1.5 \%$ & 5 & $5.4 \%$ & 5 & $11.9 \%$ \\
\hline
\end{tabular}

NVD, neovascularisation of the disc; NVE, neovascularisation elsewhere.

${ }^{\mathrm{a}}$ Missing data in 6 cases.

logMAR difference $=0.1, p<0.01$ ), but not independently related to the age of the patient, type or duration of diabetes, grade of ophthalmologist performing the laser treatment, systematic screening to detect the proliferative retinopathy or the waiting time for treatment (from listing and from the time of referral).

\section{Change in the retinopathy features}

Table 5 shows the change in the retinal new vessel features by the time of follow-up. A new vitreous haemorrhage since the first laser treatment had occurred in $17.4 \%$ (36) of patients, whilst $4.9 \%$ (10) had developed a new traction detachment and $1.5 \%$ (3) had undergone a vitrectomy. Rubeosis was present at follow-up in $2.9 \%$ (6), none of whom had had rubeosis at baseline. Where rubeosis was present at baseline $(n=10)$, this had resolved in $80 \%$ and follow-up data were not available in 2 cases. Overall, there was a poor morphological outcome as defined by rubeosis, new traction detachment, or requiring vitrectomy in $7.2 \%$ (15).

$10.5 \%$ (22) of eyes had a deterioration in the new vessel features, rubeosis, new traction detachment, or had undergone a vitrectomy by the time of follow-up. Multiple logistic regression analysis was performed to assess factors related to poor morphological outcome. Factors included in the analysis were age, gender, visual acuity at baseline, the presence of maculopathy, vitreous haemorrhage or high-risk characteristics at baseline, systematic screening to detect the retinopathy, the waiting time for the laser treatment (from listing and from referral), the grade of ophthalmologist performing the treatment, the number of laser burns given by the time of follow-up, and the area of retina treated at the first session. Poor morphological outcome (as defined by rubeosis, new traction detachment or having had a vitrectomy) was found to be related to the presence of concurrent maculopathy at baseline (OR 7.1, $p<0.01$ ), female sex (OR 7.8, $p=0.02$ ) and the presence of the equivalent of high-risk characteristics at baseline (OR 9.2, $p=0.04)$. Other factors were not found to be significantly independently related to poor morphological outcome.

For those eyes with poor outcome the initial treatment was intended to be given in one session in 4 cases. For these eyes, the time booked for the next follow-up appointment was a mean 9.7 weeks (6-13 weeks) compared with a mean 6.1 weeks (1-12) for those eyes without poor outcome $(p=0.02$, Mann-Whitney $U)$.

\section{Treatment given}

Table 6 shows the total number of laser burns given by the time of follow-up. For all cases the median number of laser burns given by the time of follow-up was 2113 (range 320-6771). There had been one treatment session in $23.8 \%$ (49), two or three sessions in $52.5 \%$ (108), four or five sessions in $21.4 \%$ (44) and six or more sessions in $2.5 \%(5)$, range $1-10$.

For those eyes whose initial treatment was intended to be given in one session, no further treatment was given in $46.2 \%$ (42) but $19.8 \%$ (18) underwent one further treatment session, $25.3 \%$ (23) underwent two further sessions, and $8.8 \%$ (8) underwent three or more treatment sessions.

For those eyes with neovascularisation of the disc (NVD) at baseline where the new vessel features had improved (regressed but still present, disappeared completely or an inactive fibrotic stalk persisted) by the time of follow-up, the median number of burns given was 2300 (range 700-6771), over a median of three

Table 6. The number of laser burns given to eyes with proliferative retinopathy by the time of follow-up

\begin{tabular}{|c|c|c|c|c|c|c|}
\hline \multirow[b]{2}{*}{ Total burns } & \multicolumn{2}{|c|}{ All cases } & \multicolumn{2}{|c|}{ Cases with 'high-risk characteristics'b } & \multicolumn{2}{|c|}{ Cases with NVD which regressed fully } \\
\hline & $n$ & $\%$ & $n$ & $\%$ & $n$ & $\%$ \\
\hline$<1000$ & 19 & $9.4 \%$ & 11 & $9.2 \%$ & 7 & $15.9 \%$ \\
\hline 1000-1999 & 72 & $35.6 \%$ & 41 & $34.2 \%$ & 15 & $34.1 \%$ \\
\hline 2000-2999 & 61 & $30.2 \%$ & 30 & $25.0 \%$ & 12 & $27.3 \%$ \\
\hline 3000-3999 & 27 & $13.4 \%$ & 20 & $16.7 \%$ & 8 & $18.2 \%$ \\
\hline 4000-4999 & 12 & $5.9 \%$ & 7 & $5.8 \%$ & 1 & $2.3 \%$ \\
\hline 5000-5999 & 8 & $4.0 \%$ & 8 & $6.7 \%$ & 0 & \\
\hline 6000-6999 & 3 & $1.5 \%$ & 3 & $2.5 \%$ & 1 & $2.3 \%$ \\
\hline Total & 202 & $100 \%$ & 120 & $100 \%$ & 44 & $100 \%$ \\
\hline
\end{tabular}

${ }^{a}$ This may represent more than one treatment session. Missing data in 7 cases.

bEyes with NVD or NVE associated with vitreous haemorrhage, broadly equivalent to high-risk characteristics as defined in the Diabetic Retinopathy Study. 
sessions (range 1-10). There was an improvement in the disc new vessels with fewer than 3000 burns in $66.6 \%$ of eyes. For those eyes with NVD prior to treatment where the new vessel features improved after just one session $(n=20)$, the median number of burns given was 1435 (range 736-2015).

For those eyes with NVD or neovascularisation elsewhere (NVE) associated with vitreous haemorrhage (broadly equivalent to the high-risk characteristics as defined in the Diabetic Retinopathy Study (DRS)), where the new vessel features had improved by the time of follow-up, the median number of burns given was 2285 (range 332-6771), given over a median of three sessions (range 1-10). For those eyes with the equivalent of DRS high-risk characteristics that improved in just one session $(n=25)$, the median number of burns given was 1404 (range 332-2414).

For eyes with rubeosis at baseline, the median number of burns to achieve regression was 3127 (range 800-5299), $n=8$.

Multiple logistic regression analysis was performed to examine factors related to the regression of new vessels. Factors included in the analysis were: age, gender, type or duration of diabetes, visual acuity at baseline, systematic screening for the retinopathy, the waiting time for the laser treatment (from listing and from referral), the presence of the equivalent of high-risk characteristics at baseline and the total number of burns given by the time of follow-up. The total number of burns given by the time of follow-up was significantly lower $(p<0.01)$ for the group in which the retinal vessels had regressed fully (either disappeared completely or an inactive fibrotic stalk persisted). The median number of burns given for those in which the new vessels had regressed was 1840 (330-6128) (over a median two sessions) compared with a median 2470 (320-6771) (over a median three sessions) in the group where the new vessels had not regressed. For those eyes whose initial treatment was to be given in one session, the area of retina treated at the first session was significantly greater in the group in which the retinal vessels had regressed fully than in the group in which retinal new vessels were persisting at the time of follow-up $(p<0.01)$. The spot size used for the initial treatment was greater in the group in which the retinal vessels had regressed $(p=0.01)$, but there was no significant difference in the number of burns used at the first treatment session.

A significantly higher proportion of eyes with just NVE at baseline achieved regression of the new vessels compared with those eyes with NVD with or without $\operatorname{NVE}(63.2 \%$ vs $38.8 \%$, chi-square $=11.2, p<0.01)$. There was no significant difference in the total number of burns given to eyes with NVE alone compared with eyes with NVD to achieve regression, or in the area of retina treated where regression occurred after just one treatment session (Mann-Whitney $U, p=0.55$ and $p=0.52$, respectively). Eyes with persistent new vessels were significantly more likely to have had a new vitreous haemorrhage by the time of follow-up than eyes in which the new vessels had regressed (chi-square $=12.7$, $p<0.001)$.

\section{Concurrent maculopathy}

For those eyes with concurrent maculopathy at baseline, this was said to have improved in $47.7 \%$ (41), not changed in $34.9 \%$ (30) and to have deteriorated in $17.4 \%$ (15). New maculopathy in the treated eye reducing the vision below $6 / 9$ had developed in $13.2 \%$ (27) of patients at follow-up.

\section{Retinopathy features in the other eye}

Proliferative retinopathy was present in the other eye in $62.3 \%$ (127) whilst there was maculopathy alone in $17.6 \%$ (36) and background retinopathy in $17.6 \%$ (36). No diabetic retinopathy had been detected in $2.5 \%$ (5). The other eye had received photocoagulation treatment in $83.3 \%$ (170).

In their better eye, $39.2 \%$ of patients had a visual acuity of $6 / 6$ or better, and $4.3 \%$ had a visual acuity of less than $6 / 60$.

\section{Discussion}

Follow-up questionnaires were returned for $85 \%$ of patients with maculopathy and $82 \%$ of patients with proliferative retinopathy, and the follow-up sample appeared to be representative of the groups at baseline. This compares favourably with the National Cataract Survey, where paired records were available on $82 \%$ of patients initially recruited. ${ }^{12}$ It is, however, of some concern that $5.4 \%$ of patients with maculopathy and $8.2 \%$ of patients with proliferative retinopathy were lost to clinical follow-up.

\section{Maculopathy}

The Early Treatment Diabetic Retinopathy Study (ETDRS) found that at 1 year follow-up, $5 \%$ of treated eyes $(n=754)$ versus $8 \%$ of untreated eyes $(n=1490)$ had lost more than 15 letters on the ETDRS chart (equivalent to $0.3 \mathrm{log}$ units or more, and representing a doubling of the visual angle). ${ }^{8}$ In this audit, $9.2 \%$ of treated eyes lost $0.3 \mathrm{log}$ units or more of visual acuity, and it would therefore appear that the results in terms of visual acuity are considerably worse than expected from the ETDRS. However, the ETDRS recruited patients without clinically significant macular oedema (39\% of the group assigned to immediate treatment) and this group could be expected to do better than eyes already affected by oedema. In our audit fewer eyes (15-19\%) did not have clinically significant macular oedema. ${ }^{11}$ In addition, eyes with other significant ocular pathology or visual acuity worse than 20/200 were excluded from the ETDRS, ${ }^{8}$ and it may well be that other ocular factors such as cataract contributed to the worse visual acuity results in our national sample. 
The finding that the follow-up visual acuity was significantly related to the baseline visual acuity is not surprising, and the finding that there is less deterioration for those eyes with worse visual acuity at baseline is almost certainly related to the fact that eyes with already poor visual acuity are not able to deteriorate much further on the scale used (recording only 'less than 6/60' for the group with the worst visual acuity). The chance of losing 0.3 log units or more of visual acuity was not found to be related to visual acuity at baseline.

The visual prognosis was better in the group with focal (vs diffuse) maculopathy, allowing for the visual acuity at baseline and treatment given as confounding variables. The visual prognosis was also better for the group given focal (vs grid) treatment, even allowing for the visual acuity at baseline and the type of maculopathy as confounding variables. The percentage of eyes suffering a drop in vision equivalent to a doubling of the visual angle was $8.5 \%$ for eyes with exudative maculopathy, $15.4 \%$ for eyes with oedematous maculopathy and $28.9 \%$ for the subgroup of eyes with diffuse oedematous maculopathy at baseline. There was an improvement in macular exudate and oedema in $77.3 \%$ and $64.6 \%$ respectively. A morphological improvement in macular exudate/oedema was found to be significantly related to better visual acuity at baseline, possibly reflecting a stage of disease that is still treatable. However, logistic regression analysis showed that an improvement in macular exudate/oedema was not independently related to the type of maculopathy or to the type of treatment given, although the visual acuity at follow-up was related to both these factors. The rates of morphological improvement would appear to be lower than in other studies, where improvement in macular exudate and oedema has been reported in $88-100 \%$. ${ }^{713}$ In this study the changes in the macular exudate or oedema were mainly based upon clinical examination, and should be treated with a degree of caution, particularly as there may have been different examiners at baseline and follow-up and the follow-up period is fairly short. The visual acuity is likely to be a more sensitive measure of outcome, since the ophthalmologist completing the questionnaire had only limited response options to the change in the maculopathy appearance (improvement, no change, a deterioration, or not known).

Waiting times (from referral and from listing) were not found to be related to the visual acuity at follow-up, but it was noted in the initial results that patients with better visual acuity at baseline waited longer to be seen in the ophthalmology clinic, suggesting a prioritisation based upon visual acuity. ${ }^{11}$ Although multiple regression analysis did not show an independent association between the visual acuity at follow-up and the detection of retinopathy by systematic screening, better visual acuity at baseline was found to be related to the detection of retinopathy by screening. ${ }^{11}$

In the ETDRS progression to high-risk retinopathy occurred in $26.7 \%$ after 5 years for those eyes with macular oedema and less severe retinopathy at baseline that were not treated with early scatter treatment. Ten per cent of eyes that did not receive scatter treatment initially had undergone scatter treatment after 1 year because of progression to early proliferative retinopathy/severe non-proliferative retinopathy (57\%) or high-risk proliferative retinopathy $(43 \%) .{ }^{14}$ The rates of progression to proliferative retinopathy are comparable in our group. In this study a higher proportion of eyes with diffuse (as opposed to focal) maculopathy developed proliferative retinopathy by the time of follow-up, although this was not found to be statistically significant $(p=0.09)$. The trend would suggest that diffuse changes may be more likely to be associated with ischaemia, which carries a greater risk of progression to neovascularisation.

In an audit of this nature, without photographs or detailed description of the morphology, it is not possible to comment on the adequacy of treatment. The treatment given in general is in accordance with expectations compared with the ETDRS, with the majority of patients with focal maculopathy being given focal treatment. It is of note, however, that grid treatment was associated with a worse visual prognosis, even allowing for visual acuity at baseline and the type of maculopathy as confounding variables.

\section{Proliferative retinopathy}

In the DRS at 8 month follow-up, $0.4 \%$ of eyes treated with argon laser had developed severe visual loss (visual acuity less than $5 / 200$ ) compared with $1.8 \%$ at 1 year. For selection into the DRS patients had to have a visual acuity of more than 20/100 at baseline. In this study, for those patients whose visual acuity at presentation was $6 / 24$ or better, $2.4 \%$ (5) had visual acuity of less than $6 / 60$ at follow-up. This is broadly comparable with the results from the DRS.

Table 7 shows a comparison of the response of NVD to treatment between this audit, a prospective study by Doft and Blankenship ${ }^{15}$ of 50 patients with proliferative retinopathy and the Diabetic Retinopathy Study. ${ }^{16}$ It can be seen that the results from this audit compare favourably with these other studies.

As expected, poor morphological outcome (defined as rubeosis at follow-up, traction detachment or having had a vitrectomy) was associated with a higher rate of 'highrisk characteristics' at baseline. Interestingly it was also associated with the presence of concurrent maculopathy at baseline. The latter may represent more severe disease, and suggest a need for more vigilant follow-up and treatment as for the conventional accepted 'high-risk characteristics'. In our audit the wait for the next followup appointment for those patients with poor morphological outcome (whose initial treatment was to be given in one session) was significantly longer than for those with better outcome. Whether the wait had contributed to the poor outcome is conjectural, but a mean wait for reassessment of 9.7 weeks is too long in the case of proliferative retinopathy, where re-intervention needs to be sooner if the response was not adequate after the first treatment. Doft and Blankenship ${ }^{15}$ found that for 


\begin{tabular}{|c|c|c|c|c|}
\hline Change to NVD & $\begin{array}{c}\text { This audit (eyes with } \\
\text { NVD alone at } \\
\text { baseline) }\end{array}$ & $\begin{array}{l}\text { Doft and Blankenship } \\
\text { (6-month follow-up } \\
\text { of eyes with } \\
\text { moderate/severe } \\
\text { NVD at baseline) }\end{array}$ & $\begin{array}{l}\text { DRS (1 year follow- } \\
\text { up of treated eyes } \\
\text { with moderate/ } \\
\text { severe NVD at } \\
\text { baseline) }\end{array}$ & $\begin{array}{l}\text { DRS (1 year follow- } \\
\text { up of untreated } \\
\text { controls) }\end{array}$ \\
\hline Full regression & $39.7 \%$ & $49 \%$ & $29.8 \%$ & $10.2 \%$ \\
\hline Partial regression & $57.3 \%$ & $23 \%$ & $24.5 \%$ & $13.1 \%$ \\
\hline Same/worse/ungradable & $3 \%$ & $28 \%$ & $45.7 \%$ & $76.8 \%$ \\
\hline
\end{tabular}

eyes undergoing panretinal photocoagulation the response of retinopathy risk factors after 3 weeks was a good predictor of the response by 6 months. A more rational time for reassessment would be at 3-4 weeks, particularly for eyes with high-risk characteristics or where one suspects that the first treatment might not have been sufficient. No other factors relating to poor outcome were ascertained, but the numbers of patients in this group was small, and it is possible that smaller associations were not detected.

The DRS recommended 800 burns of $500 \mu \mathrm{m}$ spot size as a minimum for initial panretinal photocoagulation. ${ }^{3}$ The area of retina ablated at the first treatment session was estimated using the standard formula of $\pi r^{2}$ multiplied by the number of burns. A number of different lenses would have been used in the treatments, and there may be variable uptake of laser burns, but a calculation of the maximum possible area of retina treated was made by assuming that all cases had been treated using the quadraspheric lens. The baseline data showed that some eyes were given smaller areas of retinal ablation than the minimum recommended by the DRS. ${ }^{17}$ For eyes whose initial treatment was intended to be given in one session, and which were given smaller areas of retinal ablation than the minimum recommended in the DRS, there was an improvement in the new vessel features and no further treatment was given in the follow-up period in $26.3 \%$ (10). For both eyes with NVD alone or eyes with NVD or NVE associated with vitreous haemorrhage, which improved after just one treatment session, the median area of retina ablated was $115.1 \mathrm{~mm}^{2}$, with $25 \%$ of each group receiving less than the DRS minimum (based upon a calculation assuming use of the quadraspheric lens). It would therefore appear that some eyes do respond to smaller areas of retinal ablation than recommended in the DRS protocol, including some eyes with 'high-risk characteristics'.

Those eyes in which the retinal new vessels had regressed fully had been given significantly fewer burns by the time of follow-up than those in which the vessels persisted. This will reflect the continuation of treatment for those eyes with persisting neovascularisation, and highlights the fact that some eyes respond better to treatment than others. It is certainly well documented in other studies that some eyes require more treatment than the initial DRS protocol; ${ }^{18,19}$ the lack of adequate response in some of the more heavily treated cases in our audit merely confirms the diversity of response to treatment, and the need to make allowance for individual variation.

One feature in support of giving adequate amounts of treatment at the first session was the finding that where the new vessels had regressed fully by the time of followup, and the initial treatment was given in one session, these eyes received larger areas of retinal ablation at the first treatment session than the group in which the new vessels were persisting at the time of follow-up. For these eyes the larger area of retinal ablation was found to be related to the use of larger spot size rather than the number of burns. While there may be limiting factors to achieving better results, the fact that less treatment is needed for full regression in presumably less severe cases raises the hope that if earlier detection is possible then the rate of success can be increased.

A new vitreous haemorrhage developed in $17.4 \%$ (36) of eyes after the first treatment, and the common occurrence of this complication merits a warning to the patient when obtaining informed consent in order to minimise undue anxiety. Of the 18 eyes $(7.7 \%)$ of the proliferative group that had a visual acuity of $6 / 60$ or less at follow-up, $50 \%$ were due to maculopathy and the other half had developed other complications of proliferative retinopathy either singly or in combination (i.e. from vitreous haemorrhage, traction detachment or rubeotic glaucoma). In the DRS, for eyes treated with the argon laser that did not have macular oedema at baseline, $12 \%$ lost more than 2 lines of visual acuity at 9 month follow-up. ${ }^{20}$ This compares with this audit where at follow-up $13.2 \%$ of eyes had developed new maculopathy reducing their visual acuity below 6/9.

\section{Conclusions}

Bearing in mind that audit can answer only a limited number of questions, some tentative conclusions and recommendations can be drawn:

1. For patients with maculopathy, poorer visual prognosis was related to worse visual acuity at baseline, the presence of diffuse maculopathy, and the use of grid treatment which was associated with a worse visual outcome even allowing for visual acuity at baseline and the type of maculopathy as confounding variables. These results do not support advocating the use of grid treatment to the exclusion of focal or multifocal applications. 
2. Patients with diffuse macular oedema had a greater chance of developing proliferative retinopathy and need careful follow-up to detect proliferative change.

3. For patients with proliferative retinopathy poor morphological outcome was related to the presence of high-risk characteristics, female sex, concurrent maculopathy at baseline and longer follow-up after the initial treatment. The time to the next follow-up appointment needs to be shorter than sometimes given, and a suggested time is $3-4$ weeks.

4. In view of the frequent occurrence of new vitreous haemorrhage after the first treatment, patients should be warned specifically about this possibility.

5. Our finding that for those eyes where the new vessels had regressed fully the area of retina ablated at the first session was significantly greater than for the group where the new vessels had persisted, would suggest that adequate treatment at the first session is to be recommended.

6. There is considerable variation in the response to treatment, and some eyes responded to lower or higher amounts of treatment than recommended in the DRS and ETDRS protocols. Therefore each case needs to be judged on its merit, and close follow-up is advised in case more treatment is required.

7. Although there was no apparent independent association between outcome and the detection of retinopathy by systematic screening, this study did not explore this aspect in detail. Better visual acuity at baseline for eyes with maculopathy was found to be related to the detection of retinopathy by systematic screening, and outcome was related to baseline severity for both maculopathy and proliferative retinopathy, which argues for earlier detection of retinopathy and improved screening.

We would like to thank all the ophthalmologists who took part in this audit, and made this study possible. We would also like to thank Professor D. McLeod, Mr J.S. Schilling and Mr J. Talbot for their input as members of the steering committee for this audit, Professor A.R. Rosenthal and Mr A.T. Moore as chairmen of the audit committee, and the audit secretary Mrs Janice Samson for her efficient assistance. The Department of Health provided funding for this project.

\section{References}

1. British Multicentre Photocoagulation Trial. Proliferative diabetic retinopathy: treatment with xenon-arc photocoagulation. Interim report of a multicentre randomised controlled trial. BMJ 1977;1:739-41.
2. Hercules BL, Gayed II, Lucas SB, Jeacock J. Peripheral retinal ablation in the treatment of proliferative diabetic retinopathy: a three-year interim report of a randomised, controlled study using the argon laser. Br J Ophthalmol 1977;61:555-63.

3. Diabetic Retinopathy Study Research Group. Photocoagulation treatment of proliferative diabetic retinopathy: clinical application of Diabetic Retinopathy Study findings. DRS report no. 8. Ophthalmology 1981;88:583-600.

4. Blankenship GW. Diabetic macular edema and argon laser photocoagulation: a prospective randomized study. Ophthalmology 1979;86:69-78.

5. Patz A, Schatz H, Berkow JW. Macular edema: an overlooked complication of diabetic retinopathy. Trans Am Acad Ophthalmol Otolaryngol 1973;77:34-42.

6. Townsend C, Bailey J, Kohner E. Xenon arc photocoagulation for the treatment of diabetic maculopathy. Br J Ophthalmol 1980;64:385-91.

7. Olk RJ. Modified grid argon (blue-green) laser photocoagulation for diffuse diabetic macular edema. Ophthalmology 1986;93:938-50.

8. Early Treatment Diabetic Retinopathy Study Research Group. Photocoagulation for diabetic macular edema. ETDRS report no. 1. Arch Ophthalmol 1985;103:1796-806.

9. Evans J, Rooney C, Ashwood F, Dattani N, Wormald R. Blindness and partial sight in England and Wales: April 1990-March 1991. Health Trends 1996;28:5-12.

10. Diabetes care and research in Europe: the Saint Vincent Declaration. Diabet Med 1990;7:360.

11. Bailey CC, Sparrow JM, Grey RHB, Cheng H. The National Diabetic Retinopathy Laser Treatment Audit. I. Maculopathy. Eye 1998;12:69-76.

12. Desai P. The National Cataract Surgery Survey. II. Clinical outcomes. Eye 1993;7:489-94.

13. Grey RHB. The treatment of diabetic maculopathy by argon laser photocoagulation. Trans Ophthalmol Soc UK 1986;105:424-9.

14. Early Treatment Diabetic Retinopathy Study Research Group. Early Photocoagulation for diabetic retinopathy. ETDRS report no. 9. Ophthalomology 1991;98:766-85.

15. Doft B, Blankenship G. Retinopathy risk factor regression after laser panretinal photocoagulation for proliferative diabetic retinopathy. Ophthalmology 1984;91:1453-7.

16. Diabetic Retinopathy Study Research Group. Photocoagulation treatment of diabetic retinopathy: the second report of diabetic retinopathy study findings. Ophthalmology 1978;85:82-106.

17. Bailey CC, Sparrow JM, Grey RHB, Cheng H. The National Diabetic Retinopathy Laser Treatment Audit. II. Proliferative retinopathy. Eye 1998;12:77-84.

18. Aylward GW, Pearson RV, Jagger JD, Hamilton AM. Extensive argon laser photocoagulation in the treatment of proliferative diabetic retinopathy. Br J Ophthalmol 1989;73:197-201.

19. Reddy VM, Zamora RL, Olk RJ. Quantitation of retinal ablation on proliferative diabetic retinopathy. Am J Ophthalmol 1995;119:760-6.

20. Diabetic Retinopathy Study Research Group. Macular edema in Diabetic Retinopathy Study patients. Ophthalmology 1987;94:754-60. 